\title{
EDITORIAL
}

\section{Order and disorder in the vascular network}

\author{
D Guidolin ${ }^{1}$, B Nico $^{2}$, G Mazzocchi ${ }^{1}$, A Vacca $^{3}$, GG Nussdorfer ${ }^{1}$ and D Ribatti ${ }^{2}$ \\ ${ }^{1}$ Department of Human Anatomy and Physiology, Section of Anatomy, University of Padua Medical School, Padua, Italy; \\ ${ }^{2}$ Department of Human Anatomy and Histology, University of Bari Medical School, Bari, Italy; and ${ }^{3}$ Department of Biomedical \\ Sciences and Human Oncology, University of Bari Medical School, Bari, Italy
}

Leukemia (2004) 18, 1745-1750. doi:10.1038/sj.leu.2403526

Embryonic blood vessel patterns are formed by the combined action of both deterministic and random processes. During development, an abundant capillary meshwork is formed initially and an increasing number of branches and bifurcations is generated that connect the various capillary beds to their stem vessels. ${ }^{1}$ Vascular network formation proceeds along three main stages: (i) migration and early network formation; (ii) network remodelling; and (iii) differentiation in tubular structures and the development of capillary networks, characterized by typical intercapillary distances ranging from 50 to $300 \mu \mathrm{m}$, which is instrumental for optimal metabolic exchanges. ${ }^{2}$

Several studies have been performed to understand the logic of vascular network growth. For example, a theoretical model for in vitro angiogenesis has been previously proposed ${ }^{3}$ based on experiments performed with bovine aortic endothelial cells focusing on cellular network reorganization driven by cellular traction. ${ }^{4}$ More recently, Serini et al $l^{5}$ provided a model including chemoattraction as a fundamental mechanism for cell to cell communication in order to identify key parameters in the complexity of the formation of vascular patterns. They showed that nonlinear mechanics and chemotactic cellular dynamics fit into a model able to reproduce with great accuracy the formation of capillary networks in vitro.

The chick chorioallantoic membrane (CAM) is characterized by two distinct growth periods: before incubation day 11, it expands to cover the egg from inside, but after day 11, it can expand no further. The CAM capillary network undergoes three phases of development characterized by very different growth processes. ${ }^{6}$ In the early phase (days 5-7), sprouting of new capillaries represents the dominant angiogenic mechanism. Between days 8 and 12, the capillary network expands mainly by intussusceptive microvascular growth. In the later stages (days 13 and 14), the CAM capillary network undergoes a simple expansion with not much increase in network complexity. The analysis of mitotic rates in the CAM fits well with the concept that early sprouting is followed by intussusception: mitotic rates were very high early in the CAM and decreased to very low levels after day $11 .^{6,7}$ The vascular tree of the CAM develops as a two-dimensional (2D) structure because of its confinement within the allantois, the inner layer of the bilaminar CAM. The capillary plexus develops via arteriolar/venular connections within the chorion, the outer membrane layer of the CAM, which lies adjacent to the shell.

Correspondence: Professor D Ribatti, Department of Human Anatomy and Histology, University of Bari Medical School, Policlinico, Piazza Giulio Cesare, 11, Bari I-70124, Italy; Fax: +39 0805478 310;

E-mail: ribatti@anatomia.uniba.it

Received 1 July 2004; accepted 20 August 2004
Angiogenic factors have been delivered to the CAM via encapsulation within a delivery device, such as a gelatin sponge, ${ }^{8}$ or methyl cellulose disk, ${ }^{9}$ direct placement of factorcontaining tissue or tissue-like substances (eg collagen gel) on the $\mathrm{CAM}^{10}$ or adsorption of the factor to a polymer window. ${ }^{7}$ These methods create interesting models of gradient or regional stimulation of angiogenesis. Blood vessel patterns in the chick CAM during normal development and after growth factor application have previously been characterized with measurements of vessel length density, endothelial proliferation intensity, and complexity ${ }^{7}$ and by fractal generation branching morphometry. ${ }^{11,12}$

A structural feature of particular interest in order to get a better understanding of tissue perfusion, and therefore of CAM efficiency, is the way the pattern of growing vessels fills the available space. To address this point, we have developed automatic image analysis methods allowing a quantitative evaluation of several parameters (fractal dimension, lacunarity and nonfractal order-disorder parameters, such as positional, topological and orientational order) characterizing the level of spatial order/disorder exhibited by the vascular network of the CAM in basal conditions and after treatment for $96 \mathrm{~h}$ with an angiogenic cytokine, such as fibroblast growth factor-2 (FGF-2), or with an angiostatic molecule, such as vinblastine.

At day 12, CAM's areas adjacent to the gelatin sponges soaked with $500 \mathrm{ng}$ of FGF-2 or 1 pmol vinblastine were photographed in ovo with a stereomicroscope connected to an image analysis system at a primary magnification of $\times 16$. Greylevel images were then saved as TIFF files, processed and analysed by using a computer-assisted image analysis software and macro routines specifically developed by the authors.

After brightness inversion, an adaptive discrimination procedure ${ }^{13}$ was applied to select vessel profiles virtually exclusive of all background. This method operated with a local threshold: the mean grey value of a neighbouring region was calculated for every pixel (by a $20 \times 20$ pixel low-pass filter); this value plus an offset threshold constant (40 grey levels) defined the local threshold for each pixel. A $3 \times 3$ median filter was then applied to clean the resulting binary image. By using binary thinning procedures, ${ }^{14}$ the skeleton of this image was finally derived and pruned to remove eventually present small artefactual branches.

Vascular density was estimated from the thresholded image by evaluating the area fraction $\left(A_{A}\right)$ covered by the vessels. The 'box counting' method at multiple origins ${ }^{15}$ was applied to the image of the binary skeleton to estimate fractal dimension $(D)$ and lacunarity $(L)$ of the vascular network, well-known global indices of morphological complexity and structural nonuniformity, respectively. ${ }^{16,17}$

In addition, three types of order exhibited by the CAM vessels were considered: (i) positional order, associated with the arrangement of the network in the membrane; (ii) topological 
order, associated with the homogeneity of branching occurrence along the structure; and (iii) orientational order, associated with the orientation of the branches forming the network. They were quantitatively evaluated starting from the image of the binary skeleton according to the following procedures.

Positional order: To characterize the heterogeneity in space occupancy of a 2D set of points, Voronoi's analysis was shown to be a useful tool. ${ }^{14}$ Given a spatial point pattern, the analysis starts from the calculation of a diagram (Voronoi diagram), consisting in a tessellation of the plane with cells whose sides are segments bisecting the lines linking each point with its nearest neighbours. As shown in Figure 1a, the structure of the diagram provides relevant information about the level of order/ disorder exhibited by the spatial arrangement of the points. In particular, when heterogeneity increases, the following parameter (ranging between 0 and 1 ) also increases ${ }^{18}$ :

$$
\mathrm{SD}=1-\left(1+\frac{\sigma_{A}}{\bar{A}}\right)^{-1}
$$

where $\bar{A}$ is the mean area of the Voronoi's cells and $\sigma_{A}$ their standard deviation.

This approach was applied to the set of positions formed by the branching points of the vascular tree, considered as the representative of the spatial arrangement of the network, and used to characterize its level of positional order. Branching points can be automatically identified simply by looking at the binary skeleton for the pixels with more than two neighbours. ${ }^{13}$ Voronoi diagram was then calculated by applying the Fortune's sweepline algorithm. ${ }^{19}$

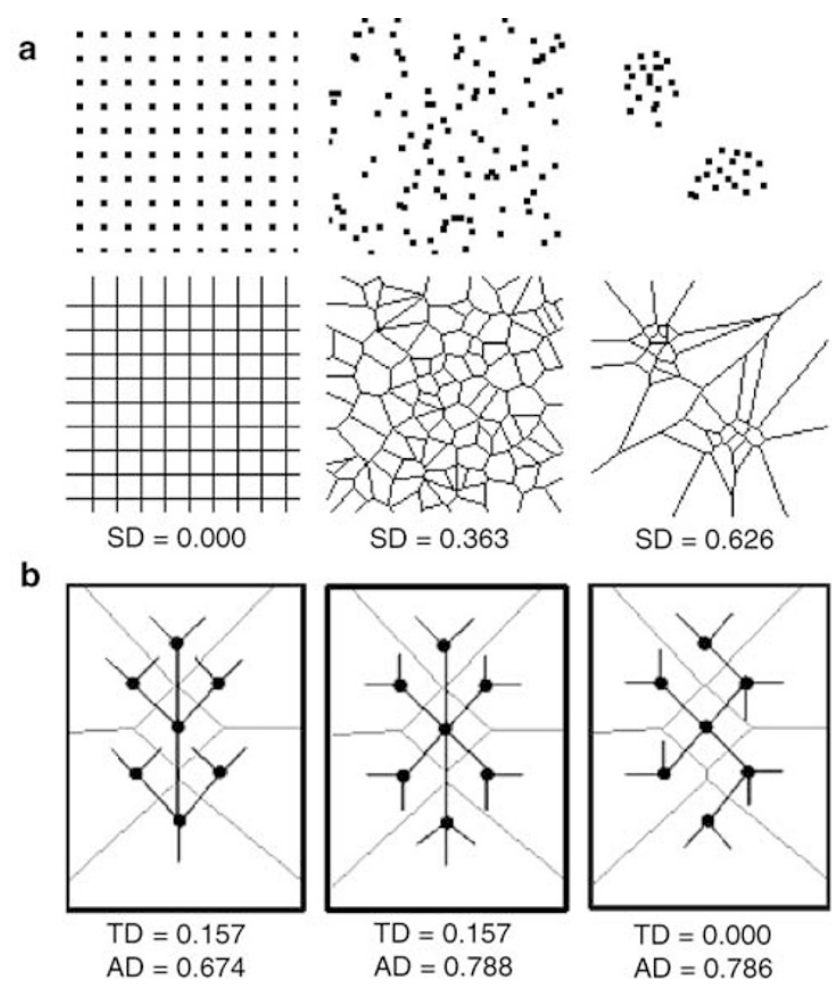

Figure 1 In (a) point patterns of increasing level of disorder together with the corresponding Voronoi diagrams and SD values. In (b) patterns characterized by the same value of SD, but differing in TD and/or AD. In particular, patterns in the left and central panel have the same TD, but they differ in AD. Those in the central and right panel have similar AD, but a different TD.
Topological order: As illustrated in Figure 1b, networks with the same size and level of positional order can be more or less irregular with respect to the occurrence of branching points along the structure. To obtain a quantitative evaluation of this morphological feature, the segments of the binary skeleton connecting the branching points were identified simply by removing from the skeleton the points with more than two neighbours. The lengths of these segments are the distances (taken along the structure) between the points where branching occurs. Therefore, indicating with $\bar{L}$ the mean length of the skeleton segments and with $\sigma_{L}$ their standard deviation, the following parameter (ranging between 0 and 1 ) was estimated:

$$
\mathrm{TD}=1-\left(1+\frac{\sigma_{L}}{\bar{L}}\right)^{-1}
$$

As shown before, this parameter increases as the heterogeneity in branching point occurrence increases.

Orientational order: Networks of similar size, positional order and pattern of branching can significantly differ with respect to the global and/or local orientation of their branches (Figure 1b). To characterize this third aspect of the 2D network arrangement, each pixel belonging to the binary skeleton was considered and its orientation estimated on the basis of the positions of its two nearest neighbours in a $3 \times 3$ mask centred on the pixel under examination. In this mask, there are eight possible directions for neighbours with respect to the central pixel, corresponding to eight angular values, which were coded as shown in Figure 2 a. Averaging the angular values of the two neighbours and warping the result in the range $0-\pi$, the orientation of the central pixel

a

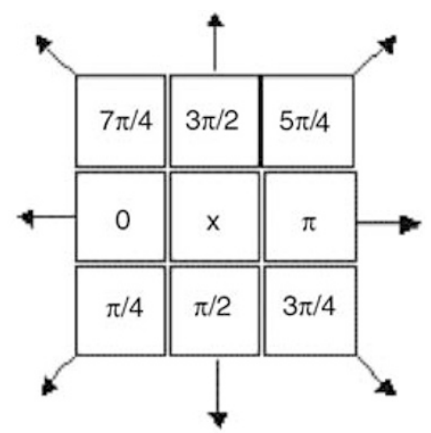

b

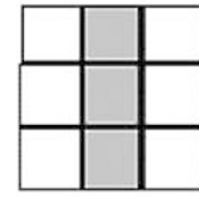

0
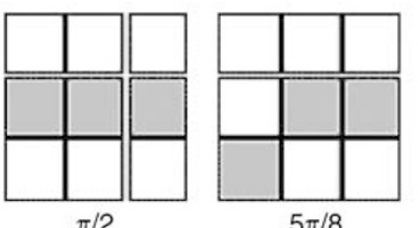

$5 \pi / 8$

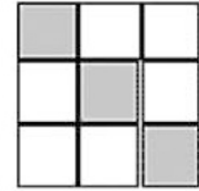

$\pi / 4$

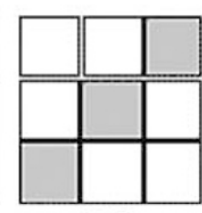

$3 \pi / 4$

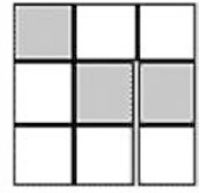

$3 \pi / 8$

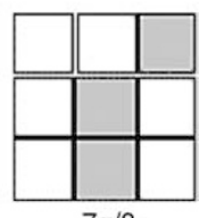

$7 \pi / 8$
Figure 2 Orientation of each pixel in the binary skeleton was calculated by a $3 \times 3$ mask (for details see text) in which the pixels surrounding the central one were coded as demonstrated in (a). Pixel configurations representative of the eight possible orientation values are shown in (b). 
(with respect to the image frame) was estimated. Owing to the discrete geometry of the digital image, following this procedure eight different orientations can be assigned to the pixels of the binary skeleton. Representative configurations are shown in Figure $2 b$.

From the distribution of the pixel among the eight possible orientations, the following parameter was calculated:

$$
\mathrm{AD}=-\frac{\sum_{i=0}^{7} p_{i} \log \left(p_{i}\right)}{\log (8)}
$$

where $p_{i}=$ (no. of pixels with orientation $\left.i\right) /($ total no. of pixels). $\mathrm{AD}$ is simply the Shannon entropy ${ }^{20}$ normalized in order to provide a result between 0 and 1 . A value of 0 corresponds to a situation where all the pixels have the same orientation, while a value of 1 indicates that the pixels are uniformly distributed among all the available orientations. An example of the application of the above procedures to the binary skeleton of a CAM vessel network is provided in Figure 3.

Results shown in Table 1 demonstrate that a significant 38\% decrease in vessel density was observed in the group treated with vinblastine as compared to the control group, while a $29 \%$ increase of the same parameter was observed after FGF-2 treatment. Moderate changes in the overall complexity were also detected in both treated groups when compared to the control one. A $8 \%$ increase of the fractal dimension was observed in the FGF-2-treated group $(P<0.05)$ and a $11 \%$ decrease in the group treated with vinblastine $(P<0.05)$.

Furthermore, the applied treatments significantly influenced the spatial arrangement of the vascular network. An increase of more than $40 \%$ in lacunarity was observed following treatment with vinblastine, indicating a more heterogeneous spatial arrangement of the CAM vessels. On the contrary, following FGF-2 treatment they result in filling the CAM in a more homogeneous way, as indicated by the significant decrease $(-31 \%)$ of this parameter with respect to the value observed in the control group.

The order-disorder parameters proposed in the present study were consistent with this finding, but they allowed a more detailed description of the changes in the spatial arrangement of the vascular tree induced by the treatment with angiogenic or angiostatic molecules. As shown in Figure 4, a significant increase in positional disorder was observed following treatment with vinblastine: SD increased by more than $30 \%$ when a
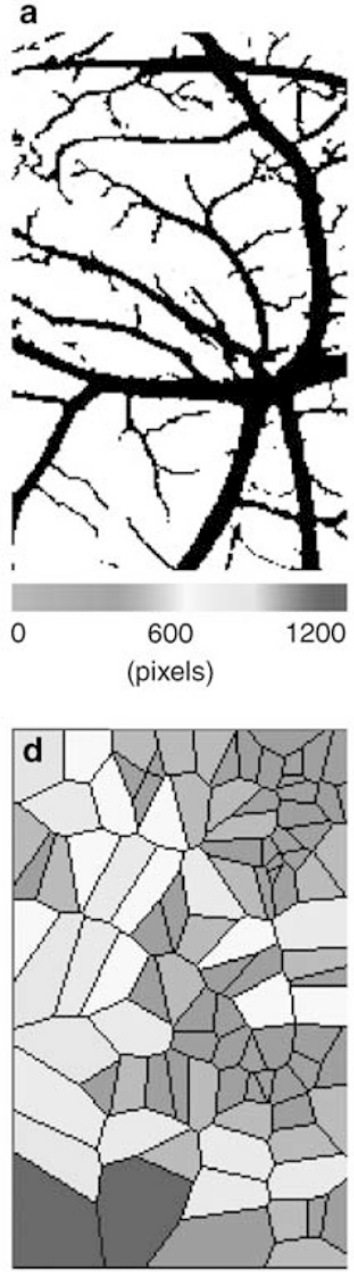

$\mathrm{SD}=0.5093$ b
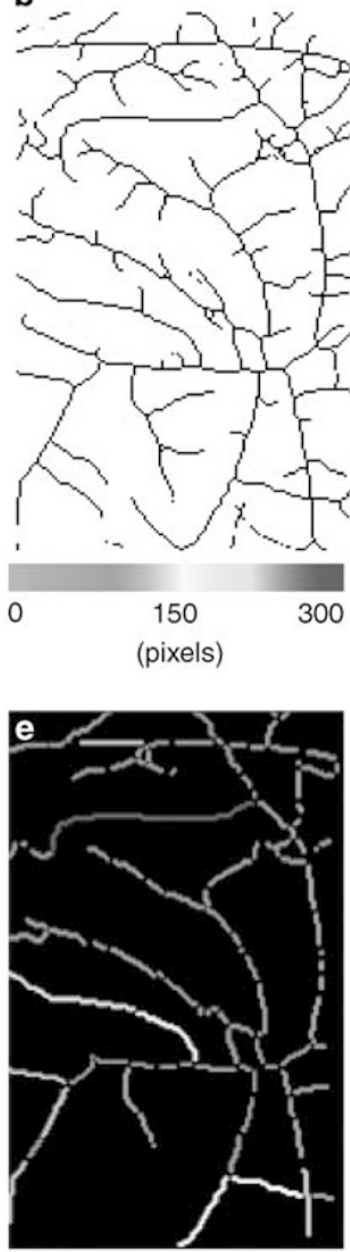

$\mathrm{TD}=0.5196$
C
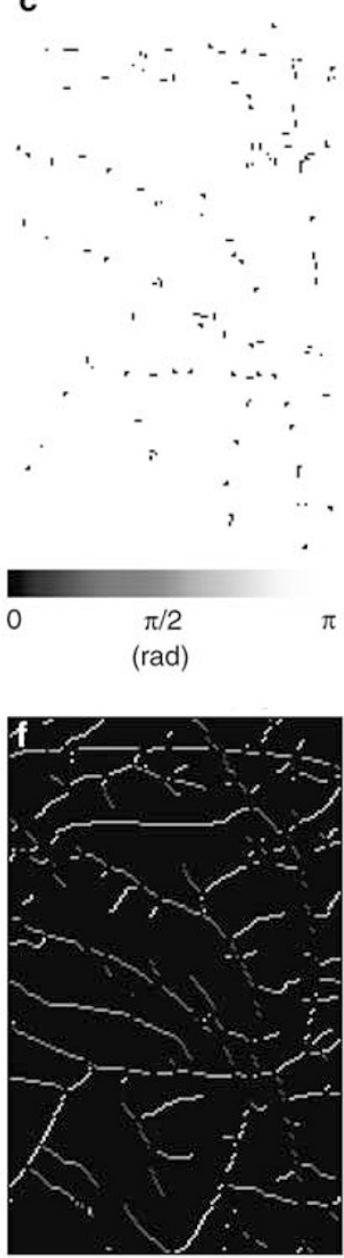

$A D=0.9063$

Figure 3 Binary image of a CAM vascular network (a) together with its binary skeleton (b) and branching points (c). The corresponding Voronoi diagram is shown in (d), where cells were colour coded according to their size. Skeleton segments connecting branching points are shown in (e) coded in colours according to their length. In (f), the pixels of the binary skeleton are shown in colours coding for their orientation. At the bottom, values of the order parameters are reported. 
Table 1 Group means ( \pm s.e.) of vascular density $\left(A_{A}\right)$, fractal dimension $(D)$ and lacunarity $(L)$

\begin{tabular}{lccc}
\hline Treatment & $A_{A}$ & $D$ & $L$ \\
\hline PBS & $0.294 \pm 0.021$ & $1.51 \pm 0.01$ & $0.288 \pm 0.02$ \\
Vinblastine & $0.181 \pm 0.020^{*}$ & $1.34 \pm 0.03^{*}$ & $0.409 \pm 0.03^{*}$ \\
FGF-2 & $0.379 \pm 0.030^{*}$ & $1.62 \pm 0.04^{*}$ & $0.198 \pm 0.02^{*}$ \\
\hline
\end{tabular}

${ }^{*} P<0.05$.
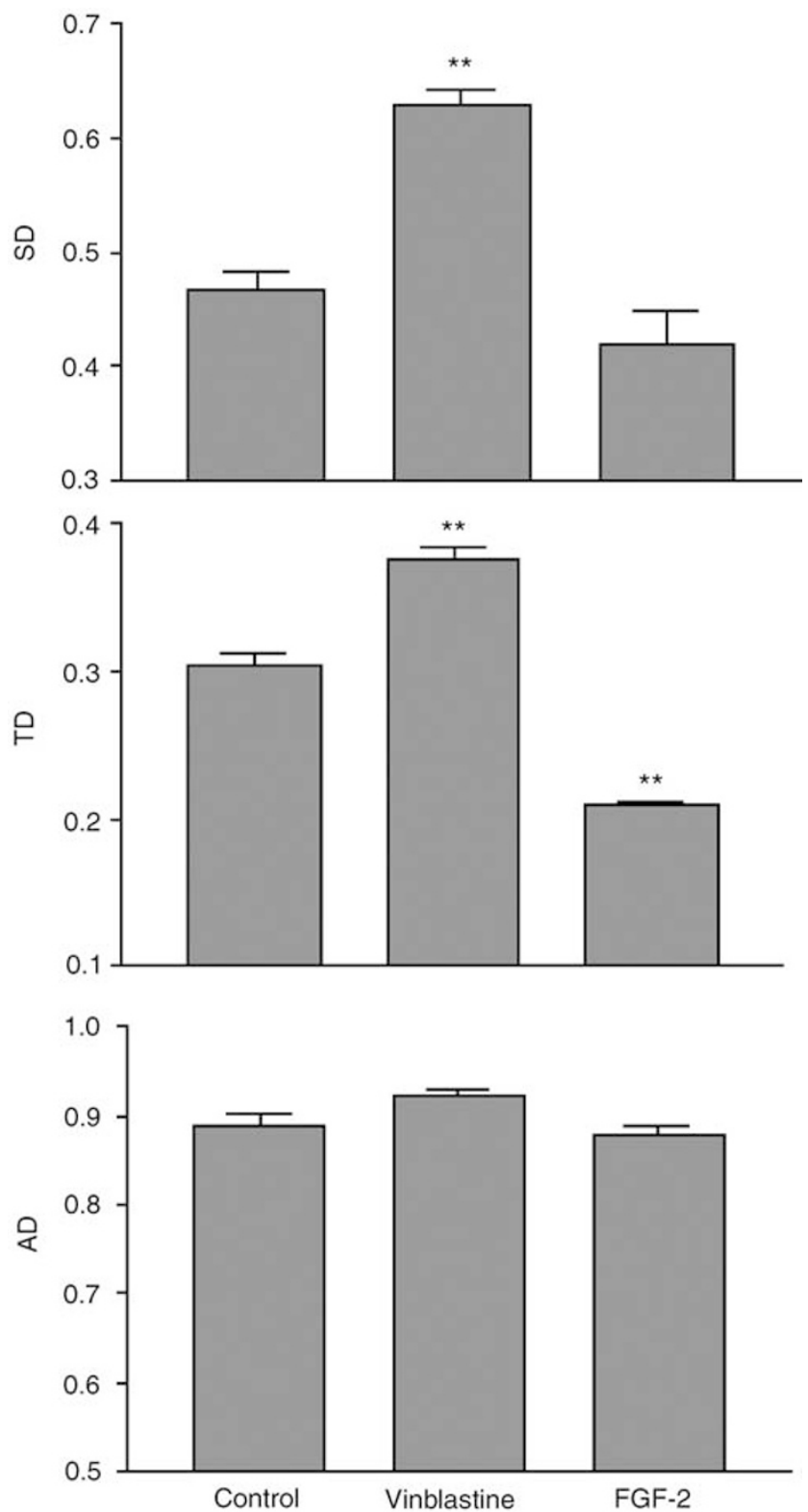

Figure 4 Order-disorder parameters in control, vinblastine- and FGF-2 treated CAMs. Values are group means \pm s.d. ${ }^{* *} P<0.05$.

compared to the value obtained in the control group. No changes in these parameters were detected following FGF-2 treatment. In this group, however, a more regular pattern of branching was observed, as indicated by the significant decrease of TD $(-31 \%)$ with respect to the control situation. On the contrary, the treatment with vinblastine led to a more irregular pattern of branching, resulting in a significant increase ( $\sim 20 \%)$ of TD. Both treatment groups did not exhibit changes in $\mathrm{AD}$ when compared with the control: at this stage of development of the vascular network, vessels were distributed among almost all the possible orientations and the observed values for $\mathrm{AD}$ were close to 1 in all groups.

The measurement of a basal rate of angiogenesis is a useful tool for the quantitative description of angiogenic perturbation by both positive and negative regulators. Blood vessel patterns in the CAM during normal development and in experimental conditions have been previously investigated with measurements of vessel length density, endothelial proliferation intensity, complexity, fractal dimension and extended counting method (XCM). $7,16,21,22$

Some of these parameters (vascular density and fractal dimension) were also used in the present study to quantitatively characterize the CAM vasculature in basal condition and after treatment with an angiogenic cyotokine, that is, FGF-2, or an angiostatic molecule, that is, vinblastine. We have previously demonstrated that FGF-2 and vinblastine are potent stimulators and, respectively, inhibitors of angiogenesis in such experimental model. ${ }^{23,24}$ The obtained results provided further evidence to that finding. As compared to the control values, while FGF-2 is responsible for an increase in vascular density and fractal dimension, vinblastine induces a decrease in vascular density and fractal dimension.

Although the growth of vessels does not seem to be fractal, ${ }^{21}$ fractal dimension, a statistical descriptor of space-filling pattern and density, was often considered as a useful statistical index characterizing the complexity of a space-filling network of vessels in studies performed by using the CAM assay. ${ }^{16,25}$ However, the vascular bed should be addressed as a highly complex structure, and fractal dimension alone may not fully characterize all the aspects of its complexity. As pointed out by Smith et al, a given value of $D$ does not uniquely specify a structure morphology and very differently looking structures can have the same or very similar fractal dimension. ${ }^{15}$ In particular, significant changes in spatial uniformity can occur without detectable changes in $D .{ }^{26}$ Parsons et al reported a significantly decreased angiogenesis in the quail CAM following treatment with either angiostatin and TGF- $\beta 1 .{ }^{11,12}$ In both studies, the decrease in $D$ with respect to the control samples appeared similar. The authors, however, observed that the application of angiostatin resulted in a disorganized arterial morphology of highly irregular spatial distribution when compared to the inhibited but normal morphology of specimens treated with TGF- $\beta 1 .^{12}$

Thus, to investigate the degree of spatial order of a structure (defined as the degree of uniformity exhibited by the structure with respect to some parameter characterizing its arrangement in the available space) other measures have to be considered besides fractal $D$. With this respect, we have tested in the present study another parameter derived from fractal geometry, that is, lacunarity. In a restrictive sense, it is a measure of the lack of rotational or translational invariance in an image. ${ }^{27}$ In a more general sense, however, it can be considered as a measure of the nonuniformity, ${ }^{15}$ potentially useful to detect situations in which irregular spatial distribution of the vascular pattern occurs as a response to angiogenic or antiangiogenic stimuli. Our data support this hypothesis. Vinblastine treatment, for instance, induced an increase in lacunarity, which was consistent with the more irregular pattern observed in treated samples when compared to the control ones. 
Lacunarity, however, as a global parameter, does not allow a detailed description of the variety of structural heterogeneities (some of them potentially significant from a biological point of view), characterizing the vascular pattern of the CAM. For this reason, we have also evaluated nonfractal order-disorder parameters, allowing a more direct description of the degree of homogeneity exhibited by the vascular structure with respect to three specific morphological features. The first one ('positional order') refers to the distribution of branching points in the membrane and could provide information about the homogeneity of development of the vascular bed in the host tissue. To evaluate this parameter, we applied Voronoi analysis. Such a geometrical tool was proven to be useful in order to detect changes in the spatial arrangement of point patterns in both biological ${ }^{18,28}$ and nonbiological ${ }^{29}$ studies. The Voronoi tessellation was also used by Kurz et $a l^{30}$ as a first approximation to model the zones of influence of each end point of a CAM vascular tree. The second structural aspect considered ('topological order') concerned the rate of occurrence of branching along an arbor structure. ${ }^{31}$ It was estimated from the lengths of the tree segments linking the branching points and it could provide some insight on how the vascular tree has grown under the influence of the applied experimental conditions. Finally, 'orientational order' was analysed to identify the possible presence of anisotropies and preferred orientations in the vessel growth.

A pattern of changes in these structural features of the vascular tree was observed following FGF-2 and vinblastine treatments. This latter induced a significant decrease in positional order and a more irregular pattern of branching, resulting in a significant increase of topological disorder. After FGF-2, the vessels resulted in filling the CAM with the same degree of homogeneity as in the control group and the positional order was not modified by the treatment. However, a significant increase of topological order was observed, expression of a more regular rate of branching during growth. Both treatments did not modify the orientational order.

In conclusion, we have demonstrated the efficacy of the CAM assay with the angiogenic inducer FGF-2 and the inhibitor vinblastine. The effects of both factors on vascular growth are clearly visible and changes in the CAM vasculature spatial arrangement relative to control specimens are readily quantified with statistical significance by using the parameters proposed here.

\section{Acknowledgements}

This work was supported in part by a grant 'Interuniversity Funds for Basic Research' (FIRB) from Ministero dell'Istruzione, dell'Università e della Ricerca, Rome (Italy) to DR and GGN.

\section{References}

1 Sandau K, Kurz H. Modelling of vascular growth processes: a stochastic biophysical approach to embryonic angiogenesis. J Microsc 1994; 175: 205-213.

2 Goldman D, Popel AS. A computational study of the effect of vasomotion on oxygen transport from capillary networks. J Theor Biol 2001; 209: 189-199.

3 Manoussaki D, Lubkin SR, Vernon RB, Murray JD. A mechanical model for the formation of vascular networks in vitro. Acta Biothereol 1996; 44: 271-282.

4 Vernon RB, Angello JC, Iruela-Arispe ML, Lane TF, Sage EH Reorganization of basement membrane matrices by cellular traction promotes the formation of cellular networks in vitro. Lab Invest 1992; 66: 536-547.

5 Serini G, Ambrosi D, Giraudo E, Gamba A, Preziosi L, Bussolino F. Modeling the early stages of vascular network assembly. EMBO J 2003; 22: 1171-1179.

6 Ausprunk DH, Knighton DR, Folkman J. Differentiation of vascular endothelium in the chick chorioallantois: a structural and autoradiographic study. Dev Biol 1974; 38: 237-248.

7 Kurz H, Ambrosy S, Wilting J, Marmé D, Christ B. Proliferation pattern of capillary endothelial cells in chorioallantoic membrane development indicates local growth control which is counteracted by vascular endothelial growth factor application. Dev Dyn 1995; 203: 174-186.

8 Ribatti D, Gualandris A, Bastaki M, Vacca A, lurlaro M, Roncali L et al. New model for the study of angiogenesis and antiangiogenesis in the chick embryo chorioallantoic membrane: the gelatin sponge/chorioallantoic membrane assay. J Vasc Res 1997; 34: 455-463.

9 Yang EY, Moses HL. Transforming growth factor beta-1 induces changes in cell migration, proliferation and angiogenesis in the chicken chorioallantoic membrane. J Cell Biol 1980; 111: 731-741.

10 Nguyen M, Shing Y, Folkman J. Quantitation of angiogenesis and antiangiogenesis in the chick embryo chorioallantoic membrane. Microvasc Res 1994; 47: 31-40.

11 Parson-Wingerter $\mathrm{P}$, Lwai B, Yang MC, Elliott KE, Milaninia A, Redlitz A et al. A novel assay of angiogenesis in the quail chorioallantoic membrane: stimulation by bFGF and inhibition by angiostatin according to fractal dimension and grid intersection. Microvasc Res 1998; 55: 201-214.

12 Parson-Wingerter $\mathrm{P}$, Elliott KE, Clark JI, Farr AG. Fibroblast growth factor-2 selectively stimulates angiogenesis of small vessels in arterial tree. Arterioscl Thromb Vasc Biol 2000; 20: 1250-1256.

13 Russ JC. The Image Processing Handbook. Boca Raton, FL, CRC Press, 1995.

14 Seul M, O'Gorman L, Sammon MJ. Practical Algorithms for Image Analysis. Cambridge: Cambridge University Press, 2000.

15 Smith TG, Lange GD, Marks WB. Fractal methods and results in cellular morphology - dimensions, lacunarity and multifractals. J Neurosci Methods 1996; 69: 123-136.

16 Kirchner LM, Schmidt SP, Gruber BS. Quantitation of angiogenesis in the chick chorioallantoic membrane model using fractal analysis. Microvasc Res 1996; 51: 2-14.

17 Guidolin D, Vacca A, Nussdorfer GG, Ribatti D. A new image analysis method based on topological and fractal parameters to evaluate the angiostatic activity of docetaxel by using the Matrigel assay in vitro. Microvasc Res 2004; 67: 117-124.

18 Marcelpoil R, Usson Y. Methods for study of cellular sociology: Voronoi diagrams and parametrization of the spatial relationships. J Theor Biol 1992; 154: 359-369.

19 Fortune SJ. A sweepline algorithm for Voronoi diagrams. Algorithmica 1987; 2: 153-174.

20 Rènyi A. On measures of entropy and information. In: Fourth Berkeley Symposium Mathematic, Statistic and Probability, Vol. 1. Berkeley: University of California Press, 1961, pp 547-561.

21 Kurz H, Wilting J, Sandau K, Christ B. Automated evaluation of angiogenic effects mediated by VEGF and PIGF homo- and heterodimers. Microvasc Res 1998; 55: 92-102.

22 Wilting J, Birkenhager R, Eichmann A, Kurz H, Martiny-Baron G, Marmè $\mathrm{D}$ et al. VEGF121 induces proliferation of vascular endothelial cells and expression of VEGF receptor-2 without affecting lymphatic vessels of the chorioallantoic membrane. Dev Biol 1996; 176: 76-85.

23 Ribatti D, Urbinati C, Nico B, Rusnati M, Roncali L, Presta M. Endogenous basic fibroblast growth factor is implicated in the vascularization of the chick embryo chorioallantoic membrane. Dev Biol 1995; 170: 39-49.

24 Vacca A, lurlaro M, Ribatti D, Minischetti M, Nico B, Ria R et al. Antiangiogenesis is produced by nontoxic doses of vinblastine. Blood 1999; 94: 4143-4155.

25 Vico PG, Kyriacos S, Heymas O, Louryan S, Cartilier L. Dynamic study of the extraembryonic vascular network of the chick embryo by fractal analysis. J Theor Biol 1998; 195: 525-532. 
26 Mandelbrot BB. A fractal's lacunarity and how it can be tuned and measured. In: Nonnenmacher TF, Losa GA, Weibel ER (eds). Fractals in Biology and Medicine. Boston: Birkhäuser, 1994, pp 8-21.

27 Allain C, Cloitre M. Characterizing the lacunarity of random and deterministic fractal sets. Physiol Rev A 1991; 44: 3552-3558.

28 Marcelpoil R, Davoine F, Robert-Nicoud M. Cellular sociology: parametrization of spatial relationships based on Voronoi diagram and Ulam trees. In: Nonnenmacher TF, Losa GA, Weibel ER (eds). Fractals in Biology and Medicine. Boston: Birkhäuser, 1994, pp 201-209.
29 Neubecker R, Schliecker G. Voronoi analysis of disorder in spontaneous optical patterns, IAP/LTO Annual Report 1998/1999, 2000, pp 31-32.

30 Kurz H, Sandau K, Wilting J, Christ B. Blood vessel growth: mathematical analysis and computer simulation, fractality and optimality. In: Mirinov V, Little C, Sage H (eds). Vascular Morphogenesis: In Vivo, In Vitro, In Mente. Boston: Birkhauser, 1997, pp 189-203.

31 Van Pelt J, Schierwagen A. Morphological analysis and modeling of neural dendrites. Math Biosci 2004; 188: 147-155. 